\title{
Многофононная внутрицентровая релаксация состояний акцепторов бора в алмазе
}

\author{
(C) Н.А. Бекин \\ Институт фризики микроструктур Российской академии наук, \\ 603950 Нижний Новгород, Россия \\ E-mail: nbekin@ipmras.ru
}

(Получена 25 апреля 2018 г. Принята к печати 7 мая 2018 г.)

\begin{abstract}
Расчеты темпов релаксации осуществлялись в адиабатическом приближении, в котором в качестве стационарных состояний примеси выступают электронно-колебательные (вибронные) состояния. Вероятности переходов между этими состояниями, сопровождаемые испусканием (или поглощением) одного или нескольких фононов, рассчитываются в первом порядке теории возмущений как возникающие в результате нарушения адиабатичности. Для описания электронной части волновой функции вибронного состояния использовался простой гамильтониан с изотропной эффективной массой. Волновая функция основного состояния находилась методом квантового дефекта. Согласно расчетам, дырка из возбужденного состояния акцептора бора, энергия которого выше энергии основного состояния на 304 мэВ, релаксирует на основное состояние с испусканием двух оптических фононов с темпом $\sim 10^{11} \mathrm{c}^{-1}$. Данная величина является оценкой сверху, поскольку использованная в расчетах модель бездисперсных оптических фононов завышает количество фононных мод, участие которых в релаксации разрешено законом сохранения энергии. Однако несмотря на грубый характер приближения, можно сделать вывод, что многофононная релаксация состояний акцепторов бора в алмазе является быстрым процессом.
\end{abstract}

DOI: $10.21883 /$ FTP.2018.11.46604.38

\section{1. Введение}

Благодаря своим уникальным свойствам алмаз является перспективным материалом для создания на его основе приборов для электроники, оптоэлектроники и квантовых вычислений $[1,2]$. Акцепторы бора - один из немногих технологически отработанных вариантов легирующей примеси в искусственном алмазе. Среди удобных для легирования примесей бор, являющийся мелкой примесью замещения, имеет наименьшую энергию связи - 370 мэВ. Интенсивные линии поглощения на примесных переходах начинаются с энергии 304 мэВ (см., например, [3]), которая существенно превышает энергию оптического фонона (165 мэВ в центре зоны Бриллюэна [4]). Таким образом, безызлучательные переходы с участием фононов с соответствующих возбужденных уровней напрямую в основное состояние являются многофононными. Физика такого рода безызлучательных переходов дырок с возбужденных уровней акцепторов интересна как с фундаментальной, так и с прикладной точек зрения.

К настоящему времени в литературе отсутствуют сведения о временах жизни состояний акцепторов бора в алмазе, как и их теоретические оценки. Имеются только скудные экспериментальные данные косвенного характера. Например, в спектрах поглощения акцепторов бора линия 304 мэВ является сильно уширенной [3]: ее ширина составляет приблизительно 6 мэВ (концентрация бора $\left.10^{17} \mathrm{~cm}^{-3}\right)$. Большая ширина линии для этого перехода может быть обусловлена быстрой релаксацией на нижележащие уровни $[3,5]$, которые, как правило, не проявляются в спектрах поглощения, поскольку соответствующие оптические переходы являются чрезвычайно слабыми. Например, линию 268 мэВ удалось обнаружить только при повышенной концентрации акцепторов [6]. Релаксация дырок возможна также при их переходах напрямую в основное состояние с испусканием, например, двух оптических фононов. Для справки приведем значения энергии (в мэВ) первых возбужденных уровней по данным обзора [5]: 240, 268, $289-$ уровни, соответствующие слабым или запрещенным (не наблюдаемым) оптическим переходам из основного состояния; 304, 337.3, 342.1 - уровни, соответствующие разрешенным оптическим переходам. Данная работа нацелена на теоретическую оценку темпа многофононной релаксации возбужденных состояний акцепторов, в стороне остается каскадный процесс релаксации дырок по промежуточным уровням, если такие имеются. Релаксация будет рассмотрена на примере уровня с энергией 304 мэВ.

Для расчета вероятностей переходов электронов (дырок) с испусканием фононов использовался подход, который рассматривает их как переходы, происходящие между собственными состояниями уравнения Борна-Оппенгеймера и вызываемые неадиабатическими членами в гамильтониане [7,8]. Этот подход позволяет рассчитывать вероятности многофононных процессов в первом порядке теории возмущений. Использование одночастотного приближения [9], в котором полагается, что все фононные моды имеют одинаковую частоту, дает возможность сделать простые оценки. Несмотря на то что такая модель является очень упрощенной, она все-таки позволяет хотя бы грубо оценить темп многофононных процессов и ответить на вопрос, существенны ли такие процессы во внутрицентровой релаксации дырок.

Метод расчета вместе с другими использованными приближениями изложен в разд. 2. Там же приведены 
результаты расчетов. В разд. 3 полученные результаты обсуждаются и делается попытка учесть известные из литературы экспериментальные данные по фактору Хуанга-Риса - важнейшего параметра теории, от которого зависит вероятность многофононного перехода. В Заключении делаются основные выводы.

\section{2. Метод расчета}

Для расчетов использовалась формула, которую получил Ридли [9], опираясь на метод, восходящий к пионерской работе Хуанга и Риса [7]. Метод основан на использовании адиабатического приближения, в котором быстрое движение частиц с легкой массой (электронов) отделяется от медленного движения тяжелых ионных остовов кристаллической решетки. Полученная в результате разделения движения электронов и решетки система описывается связанными между собой уравнениями Борна-Оппенгеймера. Эти уравнения выражают адиабатическое разделение движения электронов и фононов, однако переменные в уравнениях в строгом смысле не разделяются из-за наличия электрон-фононного взаимодействия. Таким образом, волновые функции стационарных состояний, описывающихся этими уравнениями, отвечают электронно-колебательным (вибронным) состояниям. Переходы между этими состояниями возникают вследствие нарушения адиабатичности, которое описывается дифференциальным оператором, действующим в пространстве координат решеточных (фононных) степеней свободы. Электронная часть волновой функции вибронных состояний также зависит от этих координат. Переходы между состояниями с квантовыми числами, отвечающими различным энергиям электронов, можно интерпретировать как переходы с испусканием или поглощением фононов.

В одночастотном приближении вероятность перехода из состояния $|i\rangle$ в состояние $|f\rangle$ можно записать в компактном виде:

$$
W=\omega \frac{\pi S^{p-1} e^{-S}}{(\hbar \omega)^{2}(p-1) !}\left(|\mathbf{V}|^{2} R_{0}+\frac{|\mathbf{V} \cdot \boldsymbol{\Delta}|^{2}}{S}(p-1) R_{1}\right)
$$

где

$$
\boldsymbol{\Delta}=\frac{\langle f|\mathbf{U}| f\rangle-\langle i|\mathbf{U}| i\rangle}{\sqrt{2} \hbar \omega},
$$

$\mathbf{V}=\langle f|\mathbf{U}| i\rangle, \quad S=|\boldsymbol{\Delta}|^{2}-$ фактор Хуанга-Риса, $\omega-$ частота фонона, $p=\left|E_{f}-E_{i}\right| / \hbar \omega-$ число испускаемых фононов, $E_{i}$ и $E_{f}-$ энергии начального и конечного состояний. Многомерный вектор $\mathbf{U}$ имеет количество компонент, равное количеству фононных мод в кристалле $N$, каждая из которых соответствует своему волновому вектору $\mathbf{q} ; \mathbf{q}$-компонента вектора $\{\mathbf{U}\}_{\mathbf{q}}=D_{\mathbf{q}} \exp (i \mathbf{q r})$,

$$
\left|D_{\mathbf{q}}\right|^{2}=\frac{D^{2}}{N\left(M_{r} \omega\right) / \hbar}
$$

$D$ - деформационный потенциал для оптических фононов, $M_{r}-$ приведенная масса элементарной ячейки. Формула (1) написана в низкотемпературном приближении $p^{2} \gg 4 n(n+1 / 2) S^{2}, \quad n \ll 1, \quad$ где $n=1 /[\exp (\hbar \omega / k T)-1]-$ числа заполнения фононов. Параметр $p$ можно считать непрерывной величиной [8], заменив в формуле (1) факториал гамма-функцией. Скалярные произведения в формулах подразумевают суммирование по фононным модам, которое в расчетах было заменено на интегрирование по всей зоне Бриллюэна в предположении ее сферической симметрии [10]. Входящий в оператор электрон-фононного взаимодействия $\mathbf{U}$ деформационный потенциал полагался независимым от волнового вектора $\left(D=2.1 \cdot 10^{9}\right.$ эВ/см [11]). Для вычисления зависимости безразмерных функций $R_{0}$ и $R_{1}$ от $p$ и $S$ использовались асимптотические формулы [9] при $p \gg S$.

Волновые функции $|i\rangle$ и $|f\rangle$ в формуле Ридли уже не зависят от решеточных степеней свободы, являясь решениями обычного уравнения Шредингера, зависящего только от координат электронов (дырок). Для расчетов волновых функций использовался простой изотропный гамильтониан эффективной массы: $H=p^{2} / 2 m+V_{c}(r)$, где $m$ - эффективная масса, $V_{c}(r)$ - кулоновский потенциал. Волновая функция основного состояния рассчитывалась методом квантового дефекта [10], а для возбужденных состояний химический сдвиг игнорировался. Была сделана оценка вероятности перехода дырки из состояния, энергия которого выше энергии основного состояния на 304 мэВ. Величина эффективной массы была выбрана таким образом, чтобы водородоподобные состояния с главным квантовым числом $n=2$ имели эту энергию. Это дает $m=0.63 m_{0}, m_{0}-$ масса свободного электрона. (Для сравнения, из транспортных измерений [11] для масс тяжелых и легких дырок получены соответственно следующие величины: $m_{h}=1.1 m_{0}$, $m_{l}=0.3 m_{0}$. При этом данные в литературе для эффективных масс и параметров Латтинжера имеют существенный разброс.) Для моделирования возбужденного состояния выбрана волновая функция состояния $2 p_{0}$. Заметим, что для переходов между состояниями $p$ и $s$ скалярное произведение (интеграл) $\mathbf{V} \cdot \boldsymbol{\Delta}$ в формуле (1) равно нулю, как следует из соображений симметрии.

Чтобы иметь представление о чувствительности модели к выбору частоты фонона, вероятность безызлучательного перехода представлена на рис. 1 как функция энергии фонона. В качестве ориентиров для границ разрешенных значений энергии оптических фононов (поперечных TO и продольных LO) на рисунке показаны вертикальными штриховыми линиями минимальные и максимальные значения их энергии в симметричных точках зоны Бриллюэна [4]: $\hbar \omega_{\min }^{\mathrm{TO}}=132.3 \mathrm{мэB}$, $\hbar \omega_{\min }^{\mathrm{LO}}=146.8 \mathrm{мэB}, \hbar \omega_{\max }^{\mathrm{LO} / \mathrm{TO}}=165 \mathrm{мэB}$. На рисунке приведены две зависимости, отличающиеся значением деформационного потенциала. Одна зависимость соответствует значению деформационного потенциала из работы [11] $\left(D=D_{R}=2.1 \cdot 10^{9}\right.$ эВ/см $)$, которое получено из транспортных измерений. Вторая зависимость вычислена для значения $D=D_{\text {av }}=1.4 \cdot 10^{9}$ эВ/см, которое получено с учетом экспериментального значения фактора $S$ для рассматриваемой пары уровней [3]. Способ учета 


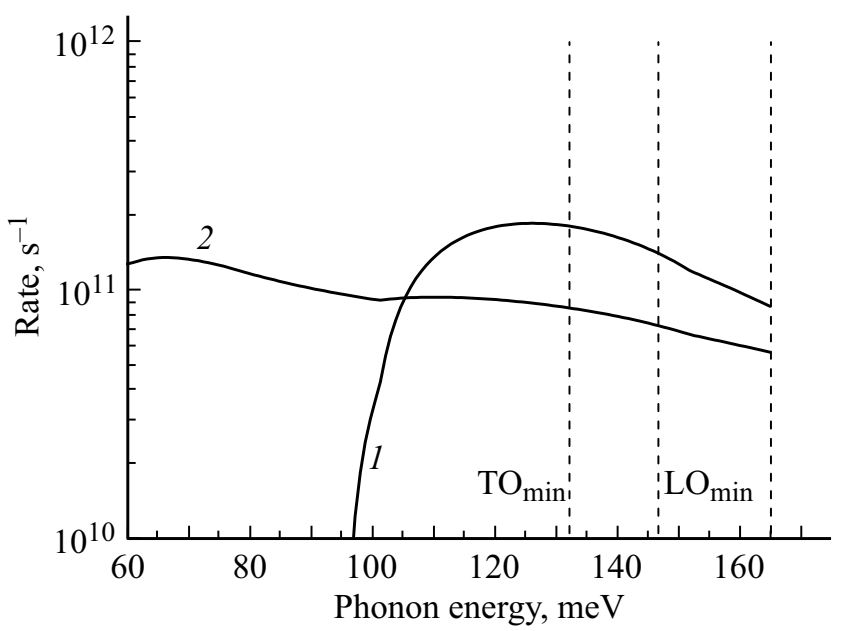

Рис. 1. Зависимость вероятности перехода из состояния с

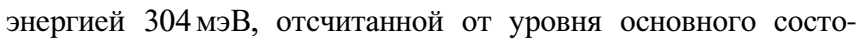
яния, в основное состояние от частоты оптического фонона как параметра модели. Деформационный потениал: 1 $D=2.1 \cdot 10^{9}{ } \mathrm{~B} / \mathrm{cm}[11], 2-D=1.4 \cdot 10^{9}{ }$ В $/$ см (с учетом экспериментальных данных по фактору Хуанга-Риса [3], объяснение в тексте). Штриховыми линиями показана разрешенная область значений для энергий фононов.

экспериментальных данных и коррекции деформационного потенциала описаны в следующем разделе.

Для обоих значений $D$, как видно из рисунка, вероятности переходов в разрешенном частотном интервале имеют порядок $10^{11} \mathrm{c}^{-1}$, что кажется нереалистично большим значением. Далее обсудим, почему использованная модель может приводить к завышению темпа релаксации.

\section{3. Обсуждение результатов и учет экспериментальных данных}

Сначала обсудим немонотонный характер зависимости вероятности безызлучательных переходов от энергии фонона как параметра модели (рис. 1). На первый взгляд, следовало ожидать, что эта зависимость должна быть монотонно возрастающей: чем больше $\hbar \omega$, тем меньше $p$ и, следовательно, тем больше вероятность процесса, поскольку в него вовлечено меньшее число фононов. Эта логика неявно подразумевает, что многофононные безызлучательные переходы электронов, которые в нулевом приближении не взаимодействуют с фононами (в обычно используемом „статическом подходе“), возникают при многочастичном электрон-фононном взаимодействии и описываются с помощью теории возмущений высоких порядков. Однако в противоположность этому для вибронных состояний электрон-фононное взаимодействие является их внутренним свойством, а испускание фононов (в том числе нескольких за один процесс) возникает при распаде вибронных состояний вследствие нарушения адиабатичности. Этот процесс, даже если он является многофононным, описывается в первом порядке теории возмущений. Рассматривая величину $p$ как формальный параметр в формуле (1), легко видеть, что в зависимости от него вероятность может в принципе принимать экстремальные значения. (Необходимо учитывать, что фактор $S$ в одночастотной модели зависит от $p$ через частоту фонона.) При $p \gg S$ и $p \gg 1$ вероятность очень быстро убывает с ростом $p$ за счет величины $(p-1) !=\Gamma(p)$ в знаменателе (1), которая возникла как статистический фактор при суммировании по фононным модам с учетом закона сохранения энергии. Таким образом, при больших $p$ характер зависимости вероятности перехода от этого параметра такой же, как при описании многофононных переходов с помощью теории возмущений высоких порядков в „статическом подходе“.

Теперь сравним полученные результаты с доступными экспериментальными данными. Как отмечалось, публикации о прямых измерениях времен жизни состояний акцепторов пока отсутствуют. С другой стороны, из эксперимента известен фактор Хуанга-Риса для рассмотренной в расчетах пары состояний [3]: $S=(0.18 \pm 0.02)$. Он был определен с использованием данных по интенсивности линий фононных повторений по отношению к интенсивности бесфононной линии 304 мэВ в спектре поглощения акцепторов бора. Линии трех фононных повторений отстояли друг от друга на 158 мэВ величину, равную энергии оптического фонона внутри разрешенной полосы (рис. 1). В одночастотной модели для указанной энергии фонона расчет дает $S=0.41$.

Учтем эти экспериментальные данные следующим образом. Заметим: для использованного в расчетах предположения о том, что деформационный потенциал не зависит от волнового вектора фононов, $S \propto D^{2}$. Используем эту зависимость для корректировки величины деформационного потенциала так, чтобы фактор $S$ совпадал с экспериментальным значением. В результате получим $D=D_{\text {av }}=1.4 \cdot 10^{9}$ эВ/см. Такая процедура коррекции предполагает, что использованная модель расчета волновых функций удовлетворительно описывает электронфононную связь вибронных состояний, а расхождение между теорией и экспериментом обусловлено неточным знанием величины деформационного потенциала. Можно предполагать, что при вычислении фактора $S$ необходимо использовать некоторое эффективное, усредненное по зоне Бриллюэна, значение деформационного потенциала $D=D_{a v}$. Оно не обязательно должно точно совпадать с величиной деформационного потенциала, полученной из транспортных измерений, когда в соответствующем усреднении играет роль окрестность центра зоны Бриллюэна. При вычислении фактора $S$ для сравнительно глубоких уровней играет существенную роль вся зона Бриллюэна.

Например, радиус локализации основного состояния в использованной модели составляет $\sim 0.4$ нм, что сопоставимо с постоянной решетки алмаза $(\sim 0.357 \mathrm{Hм})$. В связи с этим следует заметить, что описание основного 
состояния с помощью теории эффективной массы, пусть даже в скорректированном варианте метода квантового дефекта, находится под вопросом. Несомненно, что грубость в описании волновых функций может приводить к существенной погрешности для вероятности перехода. Мы, однако, будем игнорировать это обстоятельство, оставляя его учет на будущее.

Как видно из рис. 1, в интересном для нас частотном интервале коррекция деформационного потенциала не приводит к существенному понижению вероятности безызлучательных переходов. Для энергии фонона 165 мэВ коррекция приводит к понижению темпа переходов с $9 \cdot 10^{10}$ до $6 \cdot 10^{10} \mathrm{c}^{-1}$.

Из рисунка также видно, что в разрешенном частотном интервале чувствительность вероятности к энергии фонона невелика на фоне ожидаемой большой погрешности модели. Большая погрешность и явно завышенный темп многофононной релаксации, полученный с помощью описанной модели, являются платой за ее простоту. Погрешность модели возникает из двух главных источников. Во-первых, расчет электронной части волновой функции проводился в довольно грубой, электроноподобной, модели без использования специфического для описания дырок гамильтониана Латтинжера-Кона, учитывающего наличие и взаимосвязь зон легких и тяжелых дырок и спин-отщепленной зоны. Кроме того, погрешность может вносить, как отмечалось, недостаточная обоснованность применимости теории эффективной массы для описания глубоких состояний. Однако для предварительных оценок с точностью до порядка величины, на которую претендует приводимый расчет, использование простого гамильтониана является адекватным приближением. Об этом свидетельствует сопоставление теоретического и экспериментального значений фактора Хуанга-Риса: они отличаются друг от друга множителем $\sim 2$.

Другой важный источник погрешности заключается в использовании одночастотного приближения. Это упрощение должно завышать темп двухфононных переходов, что объясняется следующим образом. Процесс испускания фононов, описываемый формулой (1), является резонансным в том смысле, что фононные моды, удовлетворяющие закону сохранения энергии и дающие вклад в вероятность, равноправно распределены по всей зоне Бриллюэна: в одночастотном приближении сложение энергий любых двух фононов, принадлежащих различным модам (волновым векторам), происходит гарантированно с выполнением закона сохранения энергии. То обстоятельство, что параметр $p$ в формуле (1) может принимать нецелые значения, принципиальной роли не играет: формула, полученная при целом $p$, лишь экстраполируется на непрерывный ряд значений, поэтому резонансность процессов в описанном смысле остается. При наличии дисперсии фононов закон сохранения энергии приводит к более жесткой селекции мод, дающих вклад в вероятность безызлучательного перехода. Это справедливо и для случая $p \gg 1$, о чем свидетельствует приводимое далее сравнение результатов модели с экспериментальными данными по донорам селена в кремнии.
Рассмотрим однократно ионизованный двойной донор селена в кремнии $\mathrm{Si}: \mathrm{Se}^{+}$и релаксацию первого возбужденного состояния $1 s: T_{2}$, время жизни которого измерялось в эксперименте [12]. Энергия связи донора составляет 593 мэВ, а уровень $1 s: T_{2}$ находится выше основного состояния $1 s: A$ на 427 мэВ. С учетом того, что максимальная энергия оптического фонона составляет 62 мэВ, безызлучательные переходы на основное состояние являются существенно многофононными $(p \approx 7)$. Вычислим темп безызлучательных переходов в рамках описанной модели включая подход к вычислению волновых функций. А именно, волновую функцию основного состояния вычислим в рамках теории квантового дефекта, а волновую функцию возбужденного состояния возьмем из теории эффективной массы с учетом выбора радиуса локализации в соответствии со значением энергии состояния $1 s: T_{2}$. С учетом двукратного заряда иона радиус локализации этого состояния соответствует изотропной эффективной массе $m=1.6 m_{0}$. Следует отметить, что состояние $1 s: T_{2}$ селена возникло в результате расщепления шестикратно вырожденного (без учета спина) уровня основного состояния $1 s$. С учетом распределения огибающих волновых функций по $\Delta$-долинам оптический переход из основного состояния в $1 s: T_{2}$ является разрешенным, поэтому состояние $1 s: T_{2}$ будем моделировать антисимметричной волновой функцией состояния $2 p_{0}$. При этом мы будем игнорировать многодолинный характер волновых функций.

Предположим также, что определяющий вклад в переход дает релаксация на LO фононах с деформационным потенциалом $D=1.1 \cdot 10^{9}$ эB/см (для фонона LO-g [13]). Тогда по расчетам фактор Хуанга-Риса составляет $S=9.9$ ( $\hbar \omega=62$ мэВ). Большая величина фактора $S$ $(S \gg 1)$ соответствует сильной электрон-фононной связи, что является причиной достаточно высокой скорости многофононных процессов. При вычислении величин $R_{0}$ и $R_{1}$ (формула (1)) для случая доноров селена в кремнии, в отличие от акцепторов бора в алмазе, использовалось приближение Кондона: $R_{0}=R_{1}=1 / p^{2}$ [9]. Это связано с тем, что для релаксации состояния $1 s: T_{2}$ не выполняется условие $p \gg S$, которое было использовано ранее для расчетов $R_{0}$ и $R_{1}$. На рис. 2 показана вероятность перехода $1 s: T_{2}-1 s: A$ в зависимости от энергии фонона как параметра модели. Штриховыми линиями вновь отмечена область разрешенных энергий оптических фононов. Точкой отмечено значение вероятности перехода, соответствующее измеренному времени жизни $5.5 \cdot 10^{-9} \mathrm{c}[12]$.

Для больших $p$, в отличие от двухфононных процессов $(p \approx 2)$, появляется большая чувствительность вероятности к небольшим вариациям этого параметра. Это обусловлено, в частности, статистическим фактором $(p-1)$ ! в знаменателе формулы (1). Как видно из рисунка, из-за резкой зависимости от частоты фонона модель дает большой разброс значений темпа релаксации (на 2 порядка) внутри интересующего нас интервала. При этом экспериментальная точка лежит ниже соответствующего „доверительного интервала“ вероятностей, свиде- 


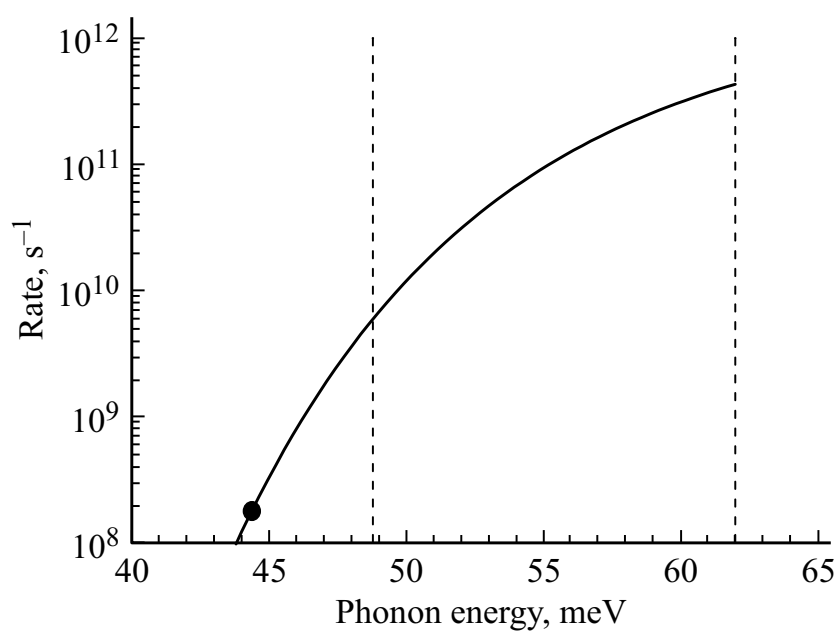

Рис. 2. Вероятность многофононной релаксации состояния $1 s: T_{2}$ однократно ионизованного двойного донора селена в кремнии $\left(\mathrm{Si}: \mathrm{Se}^{+}\right)$в зависимости от энергии фонона как параметра модели. Точкой отмечена величина темпа распада состояния согласно экспериментальным данным о его времени жизни [12]. Штриховыми линиями показана разрешенная область значений для энергий фононов.

тельствуя, что в рассмотренном примере модель дает завышенный более чем на порядок темп перехода. Вместе с тем сделанные оценки показывают, что при объяснении аномально короткого времени жизни состояния $1 s: T_{2}$ многофононные процессы могут давать существенный вклад в релаксацию, однако для получения более точных оценок темпов соответствующих переходов необходимо использовать более сложную модель, учитывающую кроме прочего дисперсию фононов.

\section{4. Заключение}

Подводя итог, можно сделать следующее заключение. Получена оценка для темпа многофононной релаксации состояния акцептора бора в алмазе с энергией 304 мэВ над основным состоянием. В рамках использованных приближений вероятность перехода имеет порядок $10^{11} \mathrm{c}^{-1}$. Данная величина является оценкой сверху, поскольку использованная в расчетах модель бездисперсных оптических фононов завышает количество фононных мод, участие которых в релаксации разрешено законом сохранения энергии. Несмотря на грубый характер приближения, можно сделать вывод, что многофононная релаксация акцепторов бора в алмазе является быстрым процессом, который может существенным образом влиять на внутрицентровую релаксацию дырок по акцепторным состояниям. Для получения более точных оценок необходимо использовать более сложный метод расчета, в частности учитывающий дисперсию фононов [8], что является предметом дальнейших исследований.

Работа выполнена при финансовой поддержке Российского фонда фундаментальных исследований (гранты РФФИ № 17-02-01325, РФФИ-ННИО № 18-502-12077).

\section{Список литературы}

[1] C.J.H. Wort, R.S. Balmer. Materials Today, 11 (1-2), 22 (2008).

[2] I. Aharonovich, E. Neu. Adv. Optical Mater., 2, 911 (2014).

[3] G. Davies, R. Stedman. J. Phys. C: Solid State Phys., 20, 2119 (1987).

[4] Semiconducrors - Basic Data, 2nd edn, ed. by O. Madelung (Springer, Berlin, 1996).

[5] J. Walker. Rep. Prog. Phys., 42, 1605 (1979).

[6] A.T. Collins, P.J. Dean, E.C. Lightowlers, W.F. Sherman. Phys. Rev., 140, A1272 (1965).

[7] K. Huang, A. Rhys. Proc. Roy. Soc. A, 204, 406 (1950).

[8] А.М. Стоунхэм. Теория дефектов в твердых телах (М., Мир, 1978) т. 1.

[9] B.K. Ridley. J. Phys. C: Solid State Phys., 11, 2323 (1978).

[10] B.K. Ridley. Solid State Electron., 21, 1319 (1978).

[11] L. Reggiani, S. Bosi, C. Canali, F. Nava, S.F. Kozlov. Phys. Rev. B, 23, 3050 (1981).

[12] K.J. Morse, R.J.S. Abraham, A.DeAbreu, C. Bowness, T.S. Richards, H. Riemann, N.V. Abrosimov, P. Becker, H.J. Pohl, M.L.W. Thewalt, S. Simmons. Sci. Adv., 3, e1700930 (2017).

[13] A. Dargys, J. Kundrotas. Handbook on physical properties of Ge, Si, GaAs and InP (Vilnius, 1994).

Редактор Л.В. Шаронова

\section{Multi-phonon intracenter relaxation of boron acceptor states in diamond}

\author{
N.A. Bekin \\ Institute for Physics of Microstructures, \\ Russian Academy of Sciences, \\ 603950 Nizhny Novgorod, Russia
}

Abstract Calculations of the relaxation rate were made in the adiabatic approximation, in which stationary states of an impurity are electron-vibrational (vibronic) states. Transition probabilities between these states, accompanied by the emission (or absorption) of one or more phonons, were calculated in the first order of perturbation theory as arising as a result of an adiabaticity disturbance. To describe the electronic part of the wave function of the vibronic state, a simple Hamiltonian with isotropic effective mass was used. The wave function of the ground state was obtained by the quantum defect method. According to the calculations, the hole from the excited state of the boron acceptor, whose energy was higher than the energy of the ground state by $304 \mathrm{meV}$, relaxed to the ground state with emission of two optical phonons at the rate of the order of $10^{11} \mathrm{c}^{-1}$. This value is an upper estimate, as used in the calculation the model of dispersionless optical phonons overestimates the number of phonon modes, whose participation in the relaxation is permitted by the law of energy conservation. However, despite the rough nature of the approximation, it can be concluded that multi-phonon relaxation of boron acceptor states in diamond is a fast process. 